Revista Arbitrada Interdisciplinaria KOINONIA

Año V. Vol V. №9. Enero - Junio 2020

Hecho el depósito de Ley: FA2016000010

ISSN: 2542-3088

FUNDACIÓN KOINONIA (F.K). Santa Ana de Coro. Venezuela.

Jimmy Alberto Caicedo-Coello; Patricio Alfredo Vallejo-Valdivieso; María Elena Moya-Martínez

http://dx.doi.org/10.35381/r.k.v5i9.617

\title{
Juegos dirigidos y la motivación en estudiantes del décimo año de Educación General Básica
}

\section{Directed games and motivation in tenth year students of Basic General Education}

\author{
Jimmy Alberto Caicedo-Coello \\ jcaicedo4577@pucem.edu.ec \\ Pontificia Universidad Católica del Ecuador, Extensión Manabí, Portoviejo \\ Ecuador \\ https://orcid.org/0000-0003-3869-9905 \\ Patricio Alfredo Vallejo-Valdivieso \\ pvallejo@utm.edu.ec \\ pvallejo@pucem.edu.ec \\ Universidad Técnica de Manabí \\ Pontificia Universidad Católica del Ecuador, Extensión Manabí, Portoviejo \\ Ecuador \\ https://orcid.org/0000-0003-3248-7864 \\ María Elena Moya-Martínez \\ mmoya@pucem.edu.ec \\ Pontificia Universidad Católica del Ecuador, Extensión Manabí, Portoviejo \\ Ecuador \\ https://orcid.org/0000-0001-7504-6933
}

Recibido: 10 de noviembre de 2019

Revisado: 20 de noviembre de 2019

Aprobado: 20 de diciembre de 2019

Publicado: 20 de enero de 2020

\section{RESUMEN}

La investigación se planteó como objetivo general: Determinar la relación entre los juegos dirigidos y la motivación en estudiantes del décimo año de Educación General Básica de la Unidad Educativa "Fanny S. de Baird" de la Parroquia Leónidas Plaza del Cantón Sucre en el primer trimestre del periodo lectivo 2018-2019. Se fundamentó desde la paradigma cuantitativo, mediante un tipo correlacionaltranseccional de Pearson, la población total fue de 127 personas participantes a quienes se les aplicó dos instrumentos tipo cuestionario en escalamiento de Likert de cuatro alternativas de repuesta con la finalidad de medir lo referido a los juegos dirigidos y motivación. Al generarse un resultado de,$- 404^{* *}$ se obtiene una correlación negativa débil, 
Jimmy Alberto Caicedo-Coello; Patricio Alfredo Vallejo-Valdivieso; María Elena Moya-Martínez

estableciéndose una relación inversamente proporcional y la motivación se ve influenciada positivamente por los juegos dirigidos, así mismo al producirse correlación significativa en el nivel 0,01 se acepta la hipótesis afirmativa y se rechaza la nula.

Descriptores: Educación alternativa; experimento educacional; innovación educacional; desarrollo de la educación. (Palabras tomadas del Tesauro UNESCO).

\section{ABSTRACT}

The research was proposed as a general objective: To determine the relationship between directed games and motivation in tenth year students of the Basic General Education at "Fanny S. de Baird" Educational Unit, in Leonidas Parish, Plaza del Cantón, Sucre in the first quarter of the 2018-2019 school period. It was based on the quantitative paradigm, through Pearson's correlation. The total population was formed by 127 participants. Two questionnaires-type instruments in Likert scaling was applied, it had four response alternatives in order to measure what was referred to directed games and motivation. When a result of,$- 404{ }^{* *}$ is generated, a weak negative correlation is obtained, establishing an inversely proportional relationship, thus being the motivation positively influenced by the directed games; likewise, when a significant correlation occurs at the 0.01 level, the affirmative hypothesis is accepted and the null is rejected.

Descriptors: Alternative education; educational experiments; educational innovations; educational development. (Words taken from the UNESCO Thesaurus)

\section{INTRODUCCIÓN}

El contexto global educativo transita en la consolidación de adopción de nuevos enfoques pedagógicos que contribuyan a una educación de calidad por medio de la innovación, es así como se ha experimentado con perspectivas educativas centradas en el estudiante (Peche Cruz \& Giraldo Supo, 2019). La centralidad en el estudiante permite proponer alternativas que en la educación tradicional no serían involucradas, siendo el juego dirigido una opción a fomentar el conocimiento en los estudiantes, para esto se requiere que el docente se encuentra formado para trabajar bajo una concepción lúdica, por cuanto el uso de juegos no implica improvisación o que se trabaja sin un objetivo establecido, al contrario, se persigue un fin de modo diverso. En este contexto, Palacio Erazo (2016), indica que: 
Jimmy Alberto Caicedo-Coello; Patricio Alfredo Vallejo-Valdivieso; María Elena Moya-Martínez

El juego es muy importante en el desarrollo de los niños y niñas, más aun cuando se trata de un juego dirigido, ya que existe la presencia de un adulto el cual se encarga de guiar la actividad y cumplir con el objetivo planteado (p. 60).

Por medio del juego dirigido, el estudiante puede aprender a desarrollar un pensamiento divergente al aprender de un modo no clásico, pudiendo generar un aprendizaje significativo a partir de los significados otorgados a un determinado objeto 0 ente de estudio, así no solo aprende, sino, que tiene la oportunidad de promover el crecimiento cognitivo a partir de una eficaz transformación de sus estructuras mentales (Martínez Albán, Vallejo Valdivieso \& Moya Martínez, 2019).Siendo indispensable que no solo estudiantes y docentes participen en el aprendizaje, es necesaria la incorporación de los padres, por cuanto el enfoque centrado en el estudiante requiere trabajar en base a un paradigma donde el discente tiene la mayor responsabilidad.

Siendo pertinente que el estudiante se encuentre motivado al aprendizaje, siendo el docente y padres un factor externo importante para lograr tal fin, considerándose además la necesidad de fomentar una motivación intrínseca. En este sentido, los juegos dirigidos son una herramienta importante en la motivación de los alumnos y alumnas, por cuanto son actividades que permiten liberar tensiones, sobre todo integrar a los miembros que se encuentran en un determinado sitio o espacio, más aun cuando esta motivación es en pro al nuevo conocimiento o el nuevo aprendizaje, por ello la motivación es sin duda alguna la clave para que el infante desee estar vinculado en los juegos, cualquiera que este sea.

Alemán Marichal, Navarro de Armas, Suárez Díaz, Izquierdo Barceló \& Encinas Alemán (2018), explican que "la motivación es un elemento importante a considerar en el proceso de enseñanza-aprendizaje. Se entiende como la intención de producir en el estudiante la ejecución consciente y deseada de una actividad" (p. 1257). Es importante reconocer el papel fundamental que tiene el juego en la motivación, más aun el dirigido por cuanto por medio de este se logra que los alumnos y alumnas fomenten sus conocimientos que traen de casa o que simplemente ya tiene, lo que se hace es que estos conocimientos se hagan significativos. Desde este punto de vista el aporte 
Jimmy Alberto Caicedo-Coello; Patricio Alfredo Vallejo-Valdivieso; María Elena Moya-Martínez teórico, el educador debe hacer reflexión sobre el papel que está ejerciendo en la formación de sus educando sobre sus conocimientos, su accionar pedagógico, aplicación de métodos innovadores que permitan motivar a sus estudiantes con juegos dirigidos que lleguen a los mismos para la formación de su nuevos conocimientos.

Así se promueve una educación dinámica, activa, recreativa, por medio de destrezas, juegos didácticos, se podría lograr que tanto estudiantes, maestros y padres de familia interactúen en el desarrollo de los conocimiento de cada uno de los estudiantes. Desde lo planteado, se contribuye además en consolidar la concepción de Buen Vivir plasmado en la Constitución de la República del Ecuador (2008), específicamente en su artículo 275. Se educa desde las posiciones que reivindican la revisión y reinterpretación de la relación entre la naturaleza y los seres humanos, manifestando que el mismo es el centro del cosmos, el hombre es único y todas las actividades humanas han sido creado por él, esta proyección del buen vivir incluye todas las actividades humanas como son las ciencias sociales, al tiempo que es novedosa.

Es así que el Ecuador viene impulsando políticas educativas que han permitido focalizar el crecimiento del país en este sector, el Instituto Nacional de Evaluación Educativa (2018), señala que:

En general, en la región latinoamericana se observa una tendencia creciente desde el 2006 al 2015. Mientras Chile encabeza la región, Ecuador se encuentra ligeramente por encima del promedio regional. En los últimos 5 años Ecuador ha mejorado en el subíndice de educación respecto de la región (pasó de estar 0,013 puntos arriba del promedio regional a estar 0,033 puntos arriba del mismo). Respecto de los países vecinos, Colombia y Perú, Ecuador comenzó en 2008. (p. 24).

La educación ecuatoriana al asumir nuevas concepciones paradigmáticas y pedagógicas enfocadas a la generación de un aprendizaje activo en el estudiante, ha logrado alcanzar metas concretas para superar progresivamente las adversidades, debilidades, del pasado, siendo pertinente contribuir desde los espacios educativos a la consolidación de las políticas educativas en cooperación desde el rol docente, por consiguiente la investigación se planteó como objetivo general: Determinar la relación entre los juegos dirigidos y la motivación en estudiantes del décimo año de Educación 
Revista Arbitrada Interdisciplinaria KOINONIA

Año V. Vol V. N9. Enero - Junio 2020

Hecho el depósito de Ley: FA2016000010

ISSN: 2542-3088

FUNDACIÓN KOINONIA (F.K). Santa Ana de Coro. Venezuela.

Jimmy Alberto Caicedo-Coello; Patricio Alfredo Vallejo-Valdivieso; María Elena Moya-Martínez

General Básica de la Unidad Educativa "Fanny S. de Baird" de la Parroquia Leónidas Plaza del Cantón Sucre en el primer trimestre del periodo lectivo 2018-2019.

\section{Aspectos teóricos}

El juego puede ser percibido como una actividad de entretenimiento o de esparcimiento, sin embargo, cuando este es dirigido con fines pedagógicos, se constituye en una estrategia que permite generar aprendizaje en el estudiante desde una óptica constructivista, Sánchez (2014), citado en Palacio Erazo (2016),opina que "la teoría constructivista se manifiesta principalmente a través de los siguientes métodos: aprendizaje-descubrimiento; aprendizaje basado en problemas; aprendizaje por indagación; aprender-haciendo" (p. 20). Al abordarse constructivistamente la educación, se puede implementar estrategias como el juego dirigido en función de promover la construcción de conocimiento a partir de la interacción entre el sujeto, sus experiencias y el objetivo que persigue el docente al trabajar en base a los juegos dirigidos. Por lo tanto, Reyes, (2014), citado en Palacio Erazo (2016), contempla que:

El juego actualmente tiene un componente estratégico esto se debe a que ambos favorecen el aprendizaje eficaz, facilitando su proceso y mejorando las capacidades y habilidades de los niños y niñas. El juego actualmente tiene un componente estratégico esto se debe a que ambos favorecen el aprendizaje eficaz, facilitando su proceso y mejorando las capacidades y habilidades de los niños y niñas (p. 20).

Lo abordado permite tener en cuenta el trabajo pedagógico en función del juego dirigido, el cual debe desarrollarse en función de los objetivos pedagógicos que procura el sistema educativo en conformidad de consolidar una educación de calidad e inclusiva, siendo necesario emplear el juego según su tipología, articulándose una relación proporcional entre el estudiante y el aprendizaje, incrementándose la capacidad metacognitiva (Muñoz Morales, Barrientos Oradini, Araya Castillo \& Reyes Saavedra, 2019). 
Revista Arbitrada Interdisciplinaria KOINONIA

Año V. Vol V. N9. Enero - Junio 2020

Hecho el depósito de Ley: FA2016000010

ISSN: 2542-3088

FUNDACIÓN KOINONIA (F.K). Santa Ana de Coro. Venezuela.

Jimmy Alberto Caicedo-Coello; Patricio Alfredo Vallejo-Valdivieso; María Elena Moya-Martínez

\section{Tipos de juegos}

Constituyen una forma de relación muy constructivista y en este sentido los trabajos a investigar se clasifican de acuerdo a las siguientes categorías, de acuerdo a AlarcónFabre (2015, p. 20):

a) Juego de descubrimiento: favorece el desarrollo intelectual a la capacidad de plantearse problema de una manera creativa y de resolver los acuerdos a la capacidad de moverse en varios estudios de desarrollo conceptual.

b) Juegos sociales: influyen en el desarrollo social y general, tiene que ver con la capacidad de al momento de suceder, realizar deseos y ambiciones capacidad imaginativa colaborar y cooperar con otras personas ayuda a la fluidez de la comunicación, aprender y valorarse así mismo.

c) Juegos imaginativos: proporciona ocasiones de expresar emociones, actuar y resolver aspectos problemáticos.

d) El juego creativo: desarrolla la destreza de coordinación pequeñas escalas, destrezas de pensamiento conceptual, abstracto lateral, repuestas creativas a problemas y capacidad para expresión y auto-expresión y la seguridad de ellos.

e) El juego colectivo: La definición que ponemos a continuación se acerca más a lo que entendemos como juego colectivo: competición mental o física desarrollada de acuerdo con unas reglas en la que los participantes juegan en oposición mutua directa y en la que cada bando trata de ganar y de impedir que lo haga el contrario.

Los juegos al ser diversos en su propósito, el docente puede diseñarlos en concordancia con los requerimientos pedagógicos plasmados en la planificación institucional, así mismo podría implementar juegos genéricos o conocidos en el ámbito educativo, así tanto docente como estudiantes ven proporcionada su creatividad, siendo este un factor que en la educación tradicional de estímulo - respuesta tiene poca cabida, por lo que la interactividad social contribuye a la conformación de un aprendizaje significativo en relación a las necesidades del estudiante. 


\section{MÉTODO}

La investigación desde una concepción metodológica se fundamentó desde la paradigma cuantitativo, mediante un tipo correlacionaltranseccional de Pearson, con la finalidad de cumplir con el objetivo general, así mismo se apoyó en lo descriptivo para identificar el comportamiento de modo aislado de las variables de estudio. Para tal propósito se trabajó con la población de estudiantes y docentes del décimo año de Educación General Básica de la Unidad Educativa "Fanny S. de Baird" de la Parroquia Leónidas Plaza del Cantón Sucre en el primer trimestre del periodo lectivo 2018-2019, la cual se encuentra conformada por 113 discentes en dos turnos de atención (mañana - tarde) y 14 docentes para una población total de 127 personas participantes a quienes se les aplicó dos instrumentos tipo cuestionario en escalamiento de Likert de cuatro alternativas de repuesta con la finalidad de medir lo referido a los juegos dirigidos (23 ítems) y motivación (27 ítems).

Ambos instrumentos fueron sometidos al juicio de tres expertos para la validación de contenido, mientras que la confiabilidad fue desarrollada mediante la aplicación de una prueba piloto aplicada a 20 sujetos con características similares a la muestra de investigación, pero que no pertenecen a la misma. El instrumento referido a juegos dirigidos obtuvo un coeficiente de Alfa de Cronbach de 0,92 mientras que el referido a motivación obtuvo un coeficiente de 0,91 lo cual los cataloga como altamente confiabilidad, empleándose el programa estadístico SPSS V25 para tal fin.

Así mismo se procedió a calcular los datos descriptivos y correlacional, con la finalidad de describir y determinar la relación de las variables de investigación, utilizándose el programa estadístico SPSS V25, permitiéndose ordenar en cuadros los resultados para su presentación y análisis, destacándose que por ser una investigación correlacionaltranseccional, la medición de información, se realizó en un único momento, los referido a lo metodológico descrito, se sustentó desde la visión de (Hernández, Fernández, Baptista, 2014). 
Revista Arbitrada Interdisciplinaria KOINONIA

Año V. Vol V. №9. Enero - Junio 2020

Hecho el depósito de Ley: FA2016000010

ISSN: 2542-3088

FUNDACIÓN KOINONIA (F.K). Santa Ana de Coro. Venezuela.

Jimmy Alberto Caicedo-Coello; Patricio Alfredo Vallejo-Valdivieso; María Elena Moya-Martínez

\section{RESULTADOS}

Se presentan los resultados de la investigación en función de dos aspectos fundamentales, el primero en describir el comportamiento de las variables desde una postura descriptiva, segundo desde una correlación en función de determinar la relación de las variables:

\section{Cuadro 1}

Uso de Juegos dirigidos en clase

\begin{tabular}{lcr}
\multicolumn{1}{c}{ Alternativas } & Frecuencia & $\%$ \\
\hline 1 Nunca & 23 & 18 \\
2 A veces & 12 & 10 \\
3 Regularmente & 13 & 10 \\
4 Siempre & 79 & 62 \\
Total & 127 & 100 \\
\hline
\end{tabular}

La opción siempre respondió en un $62 \%$ que ha participado en donde se emplea el juego dirigido en clases, mientras que las opciones regularmente $10 \%$ a veces $10 \%$ y nunca $18 \%$

\section{Cuadro 2}

Te motiva estudiar a partir del uso de juegos dirigidos

\begin{tabular}{lrr}
\multicolumn{1}{c}{ Alternativas } & Frecuencia & $\%$ \\
\hline 1 Nunca & 12 & 9 \\
2 A veces & 10 & 8 \\
3 Regularmente & 9 & 7 \\
4 Siempre & 97 & 76 \\
Total & 127 & 100 \\
\hline
\end{tabular}


Jimmy Alberto Caicedo-Coello; Patricio Alfredo Vallejo-Valdivieso; María Elena Moya-Martínez

La opción siempre respondió en un $76 \%$ que se motiva a estudiar a partir del uso de juegos dirigidos en clases, mientras que la opción regularmente se refirió en un $7 \%$ a veces $8 \%$ y nunca $9 \%$

\section{Cuadro 3}

Aprendes mejor a partir de actividades donde se juega

\begin{tabular}{lrr}
\multicolumn{1}{c}{ Alternativas } & Frecuencia & $\%$ \\
\hline 1 Nunca & 14 & 11 \\
2 A veces & 13 & 10 \\
3 Regularmente & 15 & 12 \\
4 Siempre & 85 & 67 \\
Total & 127 & 100
\end{tabular}

La opción siempre respondió en un $67 \%$ que aprende mejor a partir de actividades donde se juega, mientras que la opción regularmente se refirió en un $12 \%$ a veces $10 \%$ y nunca $11 \%$

\section{Cuadro 4}

El aprendizaje lúdico contribuye a tu formación integral

\begin{tabular}{lrr}
\multicolumn{1}{c}{ Alternativas } & Frecuencia & $\%$ \\
\hline 1 Nunca & 10 & 8 \\
2 A veces & 9 & 7 \\
3 Regularmente & 14 & 11 \\
4 Siempre & 94 & 74 \\
Total & 127 & 100 \\
\hline
\end{tabular}


La opción siempre respondió en un $8 \%$ que aprende mejor a partir de actividades donde se juega, mientras que la opción regularmente se refirió en un $7 \%$ a veces $11 \%$ y nunca $74 \%$

\section{Cuadro 5}

Correlación entre los juegos dirigidos y la motivación en estudiantes

\begin{tabular}{llrr} 
& & Juegos & Motivación \\
\hline Juegos & Correlación de Pearson & 1 &,$- 404^{* *}$ \\
& Sig. (bilateral) & & 0,000 \\
Motivación & N & 127 & 127 \\
& Correlación de Pearson &,$- 404^{* \star}$ & 1 \\
& Sig. (bilateral) & 0,000 & \\
& N & 127 & 127 \\
\hline
\end{tabular}

${ }^{* *}$. La correlación es significativa en el nivel 0,01 (bilateral).

Al generarse un resultado de -, 404 ${ }^{* *}$ se obtiene una correlación de Pearson negativa débil, estableciéndose una relación inversamente proporcional, por lo que implica que la motivación se ve influenciada positivamente por los juegos dirigidos, así mismo al producirse correlación significativa en el nivel 0,01 se acepta la hipótesis afirmativa y se rechaza la nula.

\section{Prueba de hipótesis}

Afirmativa: Existe relación significativa entre los juegos dirigidos y la motivación de los estudiantes.

Negativa: No existe relación significativa entre los juegos dirigidos y la motivación de los estudiantes. 
Revista Arbitrada Interdisciplinaria KOINONIA

Año V. Vol V. N9. Enero - Junio 2020

Hecho el depósito de Ley: FA2016000010

ISSN: 2542-3088

FUNDACIÓN KOINONIA (F.K). Santa Ana de Coro. Venezuela.

Jimmy Alberto Caicedo-Coello; Patricio Alfredo Vallejo-Valdivieso; María Elena Moya-Martínez

\section{DISCUSIÓN}

La dimensión uso de juegos dirigidos en clase al tener un 62\% de aceptación, implica que tanto estudiantes como docentes consideran satisfactoria su implementación para la promoción de una educación activa en donde lo lúdico contribuye a la conformación de un aprendizaje significativo, Córdoba, Lara \& García (2017), destacan que el juego como actividad lúdica - pedagógica permite trabajar en consecución de una educación inclusiva y de calidad para el buen vivir, siendo indispensable promover esta acción en las instituciones con la finalidad de compaginar el conocimiento con el juego, situación que permite a los estudiantes aprender en consonancia con profundizar su crecimiento integral, por cuanto se tiene en cuenta todas los factores del ser humano, como es lo cognitivo, afectivo, motor, espiritual.

La dimensión motivación a estudiar a partir del uso de juegos dirigidos, al tener una aceptación del $76 \%$ se evidencia que los estudiantes y docentes interactúanpedagógicamente para el fomento de un mejor aprendizaje, concuerdan con lo planteado porBuenahora\&Vivián Millán (2016), quienes destacan que los juegos dirigidos "favorecen el proceso de enseñanza-aprendizaje, observando mayor participación, comunicación y socialización del conocimiento entre docentes y estudiantes" (p. 50).

La dimensión aprendes mejor a partir de actividades donde se juega al tener una aceptación del $67 \%$ se encuentra en sintonía con lo descrito por Vergara Rodríguez\& Mezquita Mezquita (2016), señalan que los estudiantes perciben gratamente la implementación de juegos dirigidos con fines de aprendizaje, aunque destacan que esta debe ser combinada con clases donde se aborden las temáticas desde una concepción no lúdica, lo cual permite complementar el aprendizaje.

La dimensión formación integral a partir del aprendizaje lúdico, al tener una aceptación del $74 \%$ permite visualizar que el juego es concebido como una estrategia donde el estudiante no solo aprende los temas de clases, sino, valores, con la posibilidad de reflexionar su quehacer social en función de constituirse como ciudadano en virtud de una educación inclusiva y de calidad, Giraldo-Zuluaga (2015) indica que es necesario 
Jimmy Alberto Caicedo-Coello; Patricio Alfredo Vallejo-Valdivieso; María Elena Moya-Martínez

educar para una ciudadanía para un aprendizaje formado en la generación de una cosmovisión ética donde la persona se sienta comprometida en aportar al fortalecimiento social.

La correlación al ser significativa en el nivel 0,01 y al aceptarse la hipótesis positiva, coincide con la investigación de Hernández-Horta, Monroy-Reza \& Jiménez-García, (2018), que el estudiante se puede motivar al aprendizaje mediante la implementación de juegos, los cuales en la actualidad implica el uso de dispositivos electrónicos, el cual viene a complementar los tradicionales juegos didácticos tradicionales, lo cual implica que la educación cuenta con numerosas alternativas para propiciar un aprendizaje significativo en función de hacer uso de los diversos recursos con los cuales cuenta la sociedad globalizada en procura de promover una educación de calidad e inclusiva para el buen vivir.

\section{CONCLUSIONES}

La utilización de estrategias didácticas permite que los estudiantes sean creativos y participativos en las clases.

Las estrategias didácticas basadas en juegos dirigidos facilitan el proceso de enseñanza aprendizaje originando que los estudiantes tengan un conocimiento significativo en las distintas asignaturas.

La motivación es influenciada positivamente por los juegos dirigidos, lo cual permite motivar a los estudiantes a la consecución de un aprendizaje significativo en concordancia con su contexto social, comprendiendo que no solo se aprende para aprobar, sino, para la resolución de problemas en la vida diaria, lo cual permite crecer integralmente.

La creatividad e innovación se ve influenciada positivamente cuando se trabaja con estrategias didácticas basadas en el juego dirigido, siendo así que el estudiante tiene la oportunidad de conocerse así mismo, descubriendo talentos que sería casi imposible hacerlo en el enfoque de la educación clásica. 
Jimmy Alberto Caicedo-Coello; Patricio Alfredo Vallejo-Valdivieso; María Elena Moya-Martínez

La pedagogía lúdica se encuentra vigente, siendo necesario que los docentes y padres, conozcan más sobre el manejo de la misma con la finalidad de contribuir en un aprendizaje sustentado desde la responsabilidad del estudiante, por cuanto los nuevos enfoques educativos se basan en la centralidad del estudiante como ente responsable de su aprendizaje.

Es necesario ampliar la investigación a otros grados académicos con la finalidad de comprobar la efectividad de los juegos dirigidos en la motivación de los estudiantes, de ese modo, se podrá conocer con mayor pertinencia los alcances y debilidades de esta estrategia en función de fortalecer su alcance teórico - práctico en el sistema educativo ecuatoriano.

La investigación desarrollada se encuentra en consonancia con las políticas educativas del buen vivir del Ecuador, promoviendo además la educación inclusiva, por cuanto todos los estudiantes pueden participar en los juegos dirigidos, integrándose a un accionar social desde la educación formal.

\section{FINANCIAMIENTO}

No monetario.

\section{AGRADECIMIENTOS}

A los A los estudiantes del décimo año de Educación General Básica de la Unidad Educativa "Fanny S. de Baird" de la Parroquia Leónidas Plaza del Cantón Sucre, por su valiosa colaboración en el desarrollo de este proyecto investigativo.

\section{REFERENCIAS CONSULTADAS}

Alarcón Fabre, Y. (2015). Análisis de los juegos recreativos y su influencia en las habilidades motoras básicas de los niños de inicial I y II de educación básica en el jardín de infantes "Manuel Córdova Galarza" año lectivo 2014 -2015. [Recreational games analysis and their influence on the children's basic motor skills of $1^{\text {st }}$. and $2^{\text {nd }}$. initial education stage in the basic education of "Manuel Córdova Galarza" kindergarten, school year 2014 -2015]. Recuperado de https://n9.cl/6gip 
Revista Arbitrada Interdisciplinaria KOINONIA

Año V. Vol V. N9. Enero - Junio 2020

Hecho el depósito de Ley: FA2016000010

ISSN: 2542-3088

FUNDACIÓN KOINONIA (F.K). Santa Ana de Coro. Venezuela.

Jimmy Alberto Caicedo-Coello; Patricio Alfredo Vallejo-Valdivieso; María Elena Moya-Martínez

Alemán Marichal, B., Navarro de Armas, O., Suárez Díaz, Izquierdo Barceló, R. \& Encinas Alemán, Y. (2018). La motivación en el contexto del proceso enseñanzaaprendizaje en carreras de las Ciencias Médicas. [Motivation in the context of the teaching-learning process in Medical Sciences careers]. Recuperado de https://n9.cl/aqbl

Buenahora, M. \&Vivián Millán, L. (2016). La lúdica como estrategia pedagógica en la educación superior. [Recreational activity as a pedagogical strategy in higher education]. Recuperado de https://doi.org/10.22235/ao.v8i2.1265

Córdoba, E.F., Lara, F. \& García, A. (2017). El juego como estrategia lúdica para la educación inclusiva del buen vivir. [Game as a recreational strategy for inclusive education of good living]. ENSAYOS, Revista de la Facultad de Educación de Albacete, 32(1). Recuperado de https://n9.cl/tulyq

Giraldo-Zuluaga, G. (2015). Ciudadanía: aprendizaje de una forma de vida. [Citizenship: learning a way of life]. Educación y Educadores, 18(1), 76-92. Recuperado de https://n9.cl/4xzi

Hernández, Roberto, Fernández, Carlos, Baptista, Pilar (2014). Metodología de la Investigación (sexta edición). [Research Methodology (sixth edition)]. Editorial Mc - Graw - Hill Interamericana. México.

Hernández-Horta, Ingrid A., Monroy-Reza, Anderson, \& Jiménez-García, Martha. (2018). Learning through Games based on Principles of Gamification in Higher Education Institutions. [Aprendizaje a través de juegos basados en principios de gamificación en instituciones de educación superior]. Formación universitaria, 11(5), 31-40. https://dx.doi.org/10.4067/S0718-50062018000500031

Instituto Nacional de Evaluación Educativa (2018). La educación en Ecuador: Logros alcanzados y nuevos desafíos. Resultados educativos 2017 - 2018. [Education in Ecuador: Achievements and new challenges. Educational results 2017 - 2018]. Recuperado de https://n9.cl/g4rfm

Martínez Albán, W., Vallejo Valdivieso, P., \& Moya Martínez, M. (2019). Estructuras mentales y aprendizaje autorregulado en generación de aprendizaje significativo. [Mental structures and self-regulated learning in generating meaningful learning]. CIENCIAMATRIA, 6(10), 629-645. https://doi.org/10.35381/cm.v6i10.259

Palacio Erazo, K. (2016). El juego dirigido y el cumplimiento de consignas en los niños y niñas de primer año de la unidad educativa "Hispano América" de la ciudad de Ambato. [The directed game and the fulfillment of slogans in the first year boys and girls at "Hispano America" educational unit of Ambato city]. Recuperado de https://n9.cl/s402u 
Revista Arbitrada Interdisciplinaria KOINONIA

Año V. Vol V. N9. Enero - Junio 2020

Hecho el depósito de Ley: FA2016000010

ISSN: 2542-3088

FUNDACIÓN KOINONIA (F.K). Santa Ana de Coro. Venezuela.

Jimmy Alberto Caicedo-Coello; Patricio Alfredo Vallejo-Valdivieso; María Elena Moya-Martínez

Muñoz Morales, N., Barrientos Oradini, N., Araya Castillo, L., \& Reyes Saavedra, J. (2019). Capacidades metacognitivas en el sistema educativo en instituciones educativas de educación media. [Metacognitive capacities in the educational system in educational institutions of secondary education]. Revista Arbitrada Interdisciplinaria Koinonía, 4(7), 103-127. http://dx.doi.org/10.35381/r.k.v4i7.196

Peche Cruz, H., \& Giraldo Supo, V. (2019). El Aprendizaje Flip Learning centrado en el estudiante como generador de calidad educativa. [Student-centered Flip Learning as a generator of educational quality]. Revista Arbitrada Interdisciplinaria Koinonía, 4(8), 427-450. http://dx.doi.org/10.35381/r.k.v4i8.293

Vergara Rodríguez, D. \& Mezquita Mezquita, J. (2016). Diseño de juegos serios para reforzar conocimientos: Una experiencia educativa en secundaria. [Designing Serious Games to Reinforce Knowledge: An Educational Experience in High School]. Recuperado a partir de https://n9.cl/q2p3

(C2020 por los autores. Este artículo es de acceso abierto y distribuido según los términos y condiciones de la licencia Creative Commons Atribución-NoComercial-Compartirlgual 4.0 Internacional (CC BY-NC-SA 4.0)

(https://creativecommons.org/licenses/by-nc-sa/4.0/). 